\title{
Near-pristine gas at high redshifts: a window on early nucleosynthesis
}

\section{Max Pettini*}

Institute of Astronomy, University of Cambridge

E-mail: pettinilast.cam.ac.uk

\section{Ryan Cooke}

Institute of Astronomy, University of Cambridge, and

Department of Astronomy \& Astrophysics, University of California, Santa Cruz

E-mail: rcookeducolick.org

\begin{abstract}
It has now become recognised that damped Lyman alpha systems—gas clouds of neutral hydrogen observed in the high redshift Universe-play an important role in helping us unravel the origin of chemical elements. Here we describe the main results of a recently completed survey of the most metal-poor DLAs, aimed at complementing and extending studies of the oldest stars in the Galaxy. The survey has clarified a number of lingering issues concerning the abundances of $\mathrm{C}, \mathrm{N}$, $\mathrm{O}$ in the low metallicity regime, has revealed the existence of DLA analogues to Carbon-enhanced metal-poor stars, and is providing some of the most precise measures of the primordial abundance of Deuterium.
\end{abstract}

XII International Symposium on Nuclei in the Cosmos

August 5-12, 2012

Cairns, Australia

\footnotetext{
* Speaker.
} 


\section{Introduction}

One of the recurrent themes at this meeting has been the search for, and the study of, the most metal-poor stars in our Galaxy and its neighbours. The ultimate goal of such searches is to dig into the chemical composition of these fossil remnants for clues to the nature of the 'First Stars' which reionised the Universe and seeded it with the first generation of elements heavier than $\mathrm{Li}$.

In parallel with these efforts, we have been pursuing a complementary approach which aims to identify near-pristine pockets of gas at high redshift which may be the counterparts of the gas clouds from which the oldest Galactic stars condensed. The technique we have employed in this endeavour, called quasar (or QSO for short) absorption line spectroscopy, is illustrated in Figure 1.

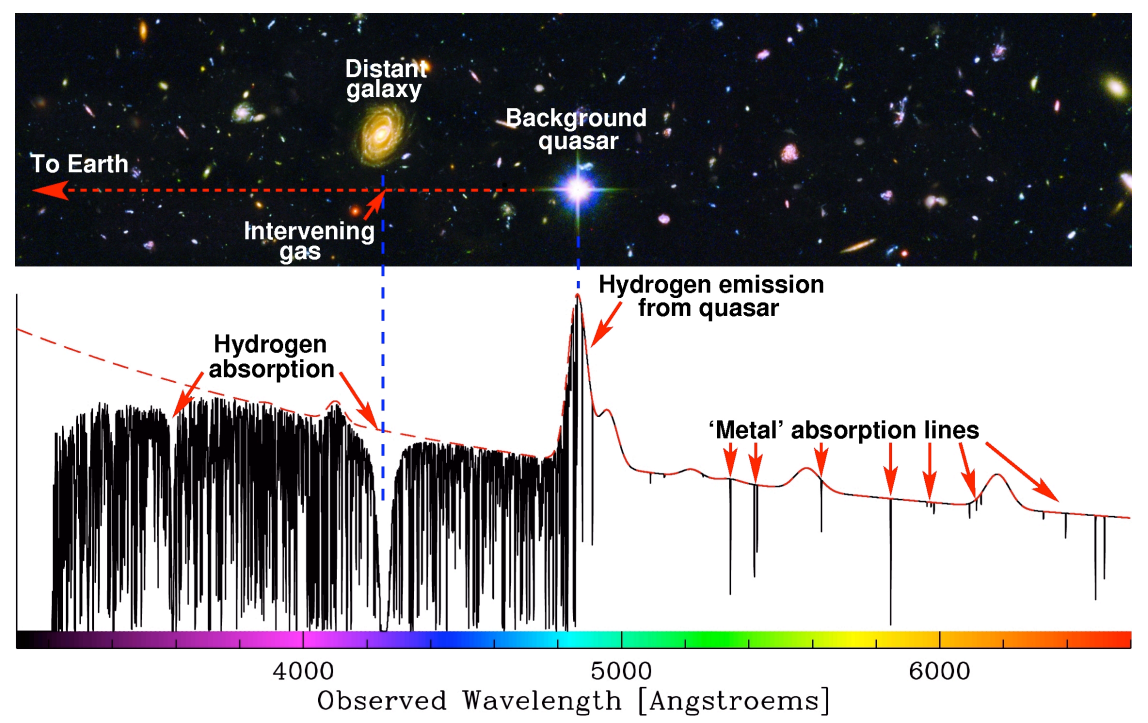

Figure 1: An illustration of the quasar absorption line technique used to probe the distant Universe (courtesy of J. Webb and M. Murphy).

QSOs are intrinsically very bright and can thus be seen to very large distances; their spectra provide a backdrop against which the absorption lines produced by intervening gas, in galaxies and the intergalactic medium, can be studied in much more detail than would otherwise be possible. Among the different types of gas clouds that can produce absorption lines in QSO spectra, we have learnt to recognise those most likely to be associated with galaxies still at an early stage of evolution - the so-called 'damped Lyman alpha systems', or DLAs for short. These systems are easily identified, even in low resolution spectra, from the strong Ly $\alpha$ absorption line at $1216 \AA$ indicative of a high column density of neutral hydrogen, $N(\mathrm{H} \mathrm{I}) \geq 2 \times 10^{20} \mathrm{~cm}^{-2}$. An example is reproduced in Figure 2. Nearly two thousand are now known thanks to large spectroscopic surveys of the sky which have been completed in the last few years, such as the Sloan Digital Sky Survey.

High resolution spectroscopy of DLAs over the last 20 years (e.g. [1] and [2] and references therein) has characterised their metallicity distribution. Most DLAs are metal-poor (unlike the brighter and more vigorously star-forming Lyman break galaxies), with typical (i.e. median) $[\mathrm{Fe} / \mathrm{H}] \simeq-1.5$. However, there is a tail in the distribution extending to $[\mathrm{Fe} / \mathrm{H}]<-3$; it is these 


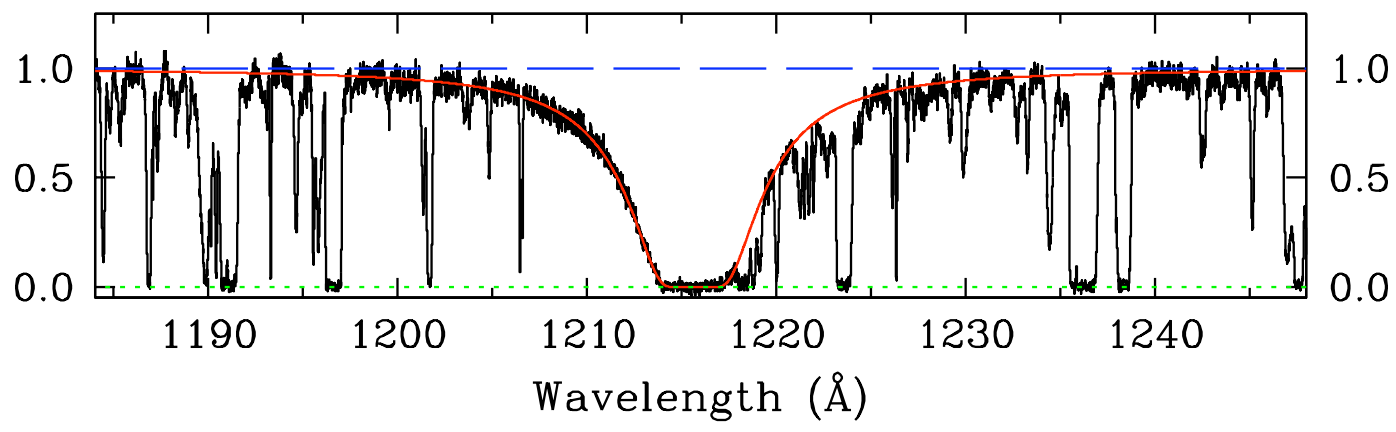

Figure 2: Example of a damped Lyman alpha absorption system. The strong Ly $\alpha$ absorption line at a rest wavelength of $1216 \AA$ (red line) is formed by high column densities of neutral gas, which are normally found in or near galaxies. Such strong spectral features are easily distinguished from the multitude of weaker absorptions due to more diffuse and ionised gas in the intergalactic medium. The $y$-axis is flux relative to the continuum of the background QSO against which the absorption is seen.

rare, least enriched DLAs that best complement and extend studies of extremely metal-poor stars in our Galaxy.

The physics of the interstellar medium is much simpler than that of stellar atmospheres. Consequently, deducing element abundances from high resolution spectra of DLAs has several advantages over its stellar equivalent. In particular, concerns about the physical conditions under which the absorption lines are formed (i.e. LTE or NLTE) do not apply to the DLAs-all the absorption lines used in abundance analyses are resonance lines from the ground states of the relevant atoms and ions. Similarly, we are not concerned with 1D vs. 3D modelling. Furthermore, when the gas is mostly neutral, as is the case in DLAs, most elements are concentrated in one dominant ionisation stage (usually either neutrals or first ions) whose ionisation potential is greater than that of hydrogen. Thus, no allowances need to be made for unseen ion stages: 'what you see is what you get'. Finally, there is the obvious attraction of carrying out these abundance measurements at high redshift, when the Universe was only $2-3$ Gyr old, and in a wider cosmological context than the parochial perspective of our Local Group.

We have recently concluded the first survey for very metal-poor DLAs [3]. Our sample is only moderately large, consisting of 22 DLAs with $[\mathrm{Fe} / \mathrm{H}]<-2$. On the other hand, with approximately one clear night on a $8-10 \mathrm{~m}$ telescope needed to secure spectra of high resolution $\left(\mathrm{FWHM} \simeq 7 \mathrm{~km} \mathrm{~s}^{-1}\right)$ and moderately high signal-to-noise ratio $(\mathrm{S} / \mathrm{N} \simeq 15-50)$ per DLA, the survey represents a considerable observational effort over a number of years. In this conference report, we highlight the most significant results uncovered by the survey. In all cases we adopt the solar abundance scale of Asplund et al. 2009 [4].

\section{Oxygen at low metallicities}

It has been known for nearly fifty years that "Moderately metal-deficient stars... have a large O/metals ratio." quoting the words of Peter Conti and collaborators in 1967 [5]. Since then, the increasingly well-documented rise in $[\mathrm{O} / \mathrm{Fe}]$ as the iron abundances decreases from solar values to $[\mathrm{Fe} / \mathrm{H}]=-1$ has been a cornerstone of Galactic chemical evolution models (e.g. [6]). As larger 

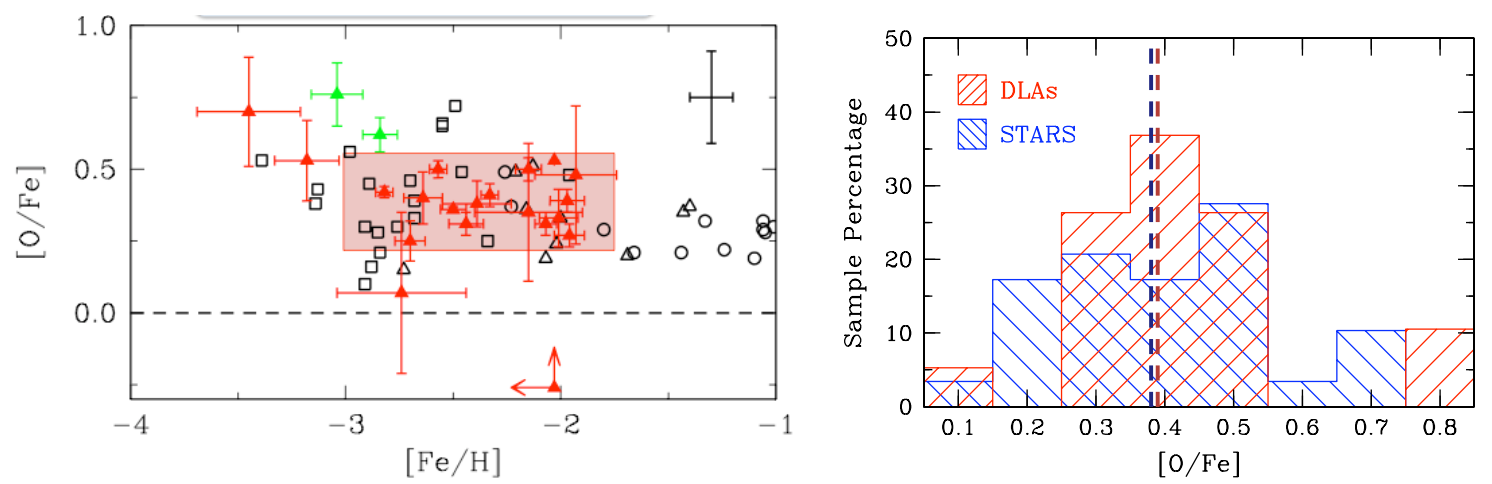

Figure 3: Left: Oxygen enhancement at low metallicities in DLAs (filled symbols) and Galactic halo stars measured from the forbidden [O I] $\lambda 6300$ line (open symbols). Original sources for the stellar measurements can be found in [3]. Right: The distributions $[\mathrm{O} / \mathrm{Fe}]$ ratios in DLAs and Galactic stars with $[\mathrm{Fe} / \mathrm{H}] \leq-2$ are very similar when values in the latter are deduced from the weak, but more reliable, [O I] $\lambda 6300$ line. The oxygen enhancement is relatively modest, $[\langle\mathrm{O} / \mathrm{Fe}\rangle] \simeq+0.4$ in both high redshift gas clouds and in local, old stars.

telescopes brought within the reach of high resolution spectroscopy stars of lower and lower metallicity, a debate has ensued as to whether the increase in $[\mathrm{O} / \mathrm{Fe}]$ with decreasing $[\mathrm{Fe} / \mathrm{H}]$ continues monotonically to reach values as high as $[\mathrm{O} / \mathrm{Fe}] \simeq+1$, or settles on a roughly constant plateau at a more modest $[\mathrm{O} / \mathrm{Fe}] \simeq+0.4$. The controversy is due in part to differing values of the oxygen abundance deducded from the analysis of different spectral features, each suffering to different degrees from (at times uncertain) corrections for NLTE and 3D effects.

These issues have now been largely settled by measurements of $[\mathrm{O} / \mathrm{Fe}]$ in very metal-poor DLAs. As can be seen from Figure 3, in DLAs with $[\mathrm{Fe} / \mathrm{H}]=-2$ to -3 , the $[\mathrm{O} / \mathrm{Fe}]$ ratio is relatively uniform around a moderate enhancement: $[\langle\mathrm{O} / \mathrm{Fe}\rangle]=+0.35 \pm 0.09$. This mean value is in good concordance with those found in old stars of the Milky Way within the same metallicity interval, provided the oxygen abundance is deduced from the weak [O I] $\lambda 6300$ line. Among the various $\mathrm{O}$ spectral features targeted in the metal-poor regime, this is the line which forms in LTE and is subject to only modest $3 \mathrm{D}$ corrections.

Does the $[\mathrm{O} / \mathrm{Fe}]$ ratio remain constant at the above value when the metallicity is lower than $1 / 1000$ of solar, or does it climb to more pronounced enhancements? This is an important question, closely linked to the initial mass function (IMF) of the first few generations of stars. Unfortunately, we do not have an answer yet. There are very tentative indications of a further increase (see Figure 3), but the number of known DLAs with such extremely low metallicities is still very small, while the stellar [O I] $\lambda 6300$ line becomes too weak to be measured reliably with current instrumentation. Both obstacles will undoubtedly be overcome in the not too distant future, so watch this space!

\section{Carbon at low metallicities}

The relative abundances of carbon and oxygen exhibit a complex behaviour as a function of metallicity (in this case measured via $\mathrm{O} / \mathrm{H}$ ). Referring to Figure 4, it can be seen that in Galactic stars $\mathrm{C}$ becomes progressively less abundant than $\mathrm{O}$ as the $\mathrm{O}$ abundance drops from solar to $1 / 10$ 


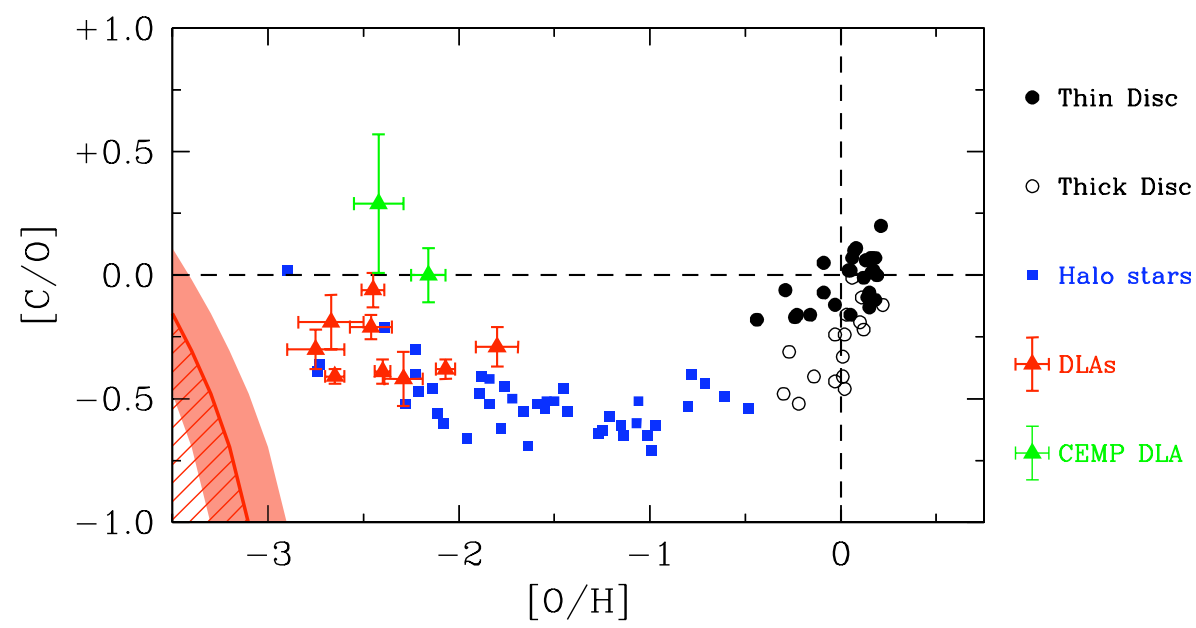

Figure 4: Carbon and oxygen abundances in DLAs and Galactic stars as indicated. Original sources for the stellar measurements can be found in [3].

solar; at $[\mathrm{O} / \mathrm{H}] \simeq-1,[\mathrm{C} / \mathrm{O}] \simeq-0.5$. This behaviour, which has been known for a while, is thought to arise from the combination of two effects: the reduced mass loss from massive stars at lower metallicities and the delayed production of $\mathrm{C}$ from the late stages in the evolution of intermediateand low-mass stars (e.g. [7]).

What had not been appreciated until relatively recently is that, as we move to even lower metallicities, the $\mathrm{C} / \mathrm{O}$ ratio appears to slowly recover, reaching again near-solar proportions when $[\mathrm{O} / \mathrm{H}] \sim-3$ (see Figure 4). This behaviour, first seen in metal-poor halo stars by Akerman et al. [7], was totally unexpected - without an additional source of $\mathrm{C}$ at the lowest metallicities, the $\mathrm{C} / \mathrm{O}$ ratio was predicted to plummet when $[\mathrm{O} / \mathrm{H}] \lesssim-2$. For this reason, Akerman et al. 'hedged their bets', by flagging the possibility that the trend may not be real, but be caused by the increasing importance with decreasing metallicity of NLTE corrections to the C I and O I lines used in their abundance determinations. Such concerns have now been largely assuaged by our finding a good agreement between the $[\mathrm{C} / \mathrm{O}]$ values measured in DLAs and Galactic stars with $[\mathrm{O} / \mathrm{H}]<-2$. A concerted analysis of the NLTE corrections [8] has also confirmed that the stellar rise is indeed real.

It thus seems to be reasonably well established that at the lowest metallicities stellar nucleosynthesis can produce copious amounts of $\mathrm{C}$, a conclusion which we suspect must be in some way linked to increasing proportion of C-enhanced metal-poor (CEMP) stars at the lowest metallicities. This topic has been discussed extensively at this meeting (see contributions by Carlo Abate, Wako Aoki, and Catherine Kennedy). Of particular relevance to our understanding of the nature of CEMP stars is the discovery of one, or possibly two, CEMP DLAs in the course of our survey [9], [10]. In the DLAs, the C-enhancement presumably reflects the overall chemical composition of the gas from which subsequent generations of stars formed, rather than being the result of masstransfer from an unseen companion. Thus, these rare CEMP DLAs may well be the high-redshift counterparts of the CEMP-no stars found in the halo of the Galaxy and its companions. Again, the connection will undoubtedly be clarified as more metal-poor DLAs are identified and targeted with high resolution spectroscopy. 


\section{Nitrogen at low metallicities}

With two UV triplets available and an ionisation potential close to that of $\mathrm{H}, \mathrm{N}$ is an element whose abundance is relatively easy to measure in DLAs. This is fortunate given the difficulty of analogous measurements in metal-poor stars and the lack of $\mathrm{H}$ II regions (the other source of $\mathrm{N}$ abundance determinations) with metallicities below $1 / 30$ of solar. Thus, it is thanks to DLAs that we have been able to probe the nucleosynthesis of $\mathrm{N}$ in the very metal-poor regime (Figure 5).

$\mathrm{N}$ and $\mathrm{O}$ Abundances in $\mathrm{H}$ II regions and DLAs

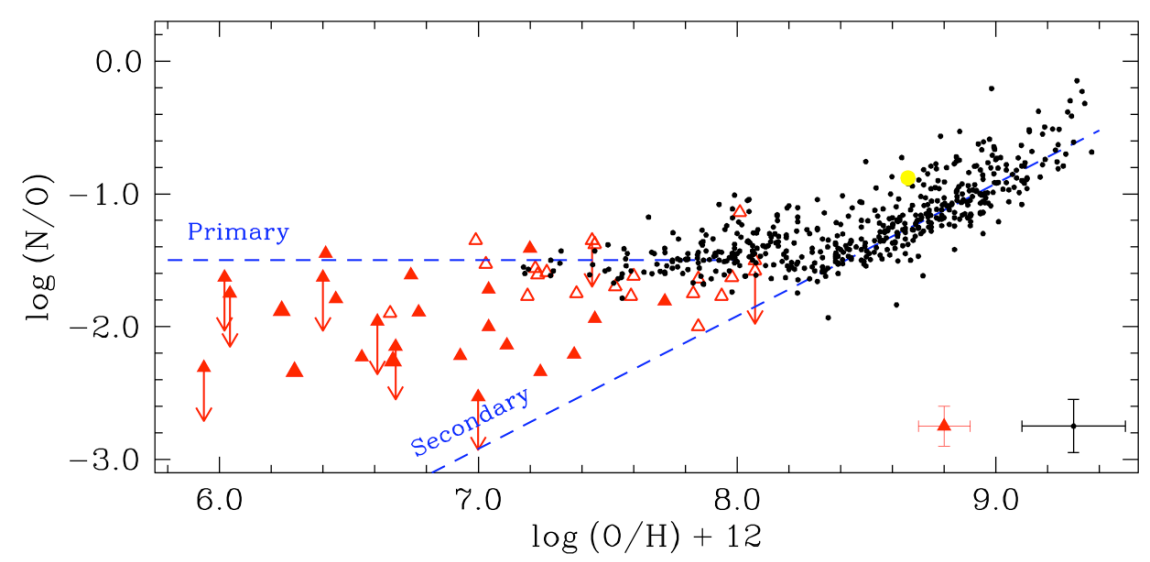

Figure 5: Nitrogen and oxygen abundances in DLAs (triangles) and nearby H II regions (small dots). Filled triangles denote DLAs in which $\mathrm{O} / \mathrm{H}$ could be measured directly, while open triangles are cases where the available $\mathrm{O}$ I lines are saturated and the oxygen abundance was deduced from $\mathrm{S} / \mathrm{H}$, assuming a solar $\mathrm{S} / \mathrm{O}$ ratio. Original sources for the H II region measurements can be found in [10]. The error bars in the bottom right-hand corner give an indication of the typical uncertainties. The large yellow dot shows the solar abundances of the two elements.

In a plot of $\log (\mathrm{N} / \mathrm{O})$ vs. $\log (\mathrm{O} / \mathrm{H})$, metal-poor DLAs fall between the boundaries set by the primary and secondary production of $\mathrm{N}$. The two terms denote the synthesis of $\mathrm{N}$ from seed $\mathrm{C}$ and $\mathrm{O}$ which are either manufactured by the star during He burning (primary $\mathrm{N}$ ), or were already present when the star first condensed out of the interstellar medium (ISM; secondary N). This is qualitatively in agreement with the idea (e.g. [12]) that the main sources of primary $\mathrm{N}$ are intermediate-mass stars on the asymptotic giant branch, such that the release of nitrogen into the ISM takes place some time after the massive stars which are the main producers of $\mathrm{O}$ have exploded as Type II supernovae.

A few additional points are noteworthy. First, the finding that the DLA measures are spread approximately uniformly between the primary and secondary boundaries suggests that the release of $\mathrm{N}$ into the ISM takes place over longer timescales than the few $10^{8}$ years normally considered between the release of $\mathrm{N}$ and $\mathrm{O}$, unless we observe most DLAs at a special time, which seems unlikely (recall that the age of the Universe at redshifts $z=2-3$, where most DLAs are observed, is $\sim 2-3 \mathrm{Gyr}$ ). In any case, the survey results dispel the idea of a bi-model distribution in the values of N/O which had been mooted on the basis of smaller samples of metal-poor DLAs [13]. Second, there seems to be a 'floor' to the minimum value of the $\mathrm{N} / \mathrm{O}$ ratio at $\log (\mathrm{N} / \mathrm{O}) \simeq-2.3$. In most cases, this is close to the limit of the DLA measurements, so that it is unclear whether the 'floor' 
simply reflects the sensitivity of our current instruments or is a real minimum value of the relative abundances of these two elements. If the latter were true, the simplest interpretation would be that this is primary $\mathrm{N}$ manufactured by massive stars, a much debated possibility.

\section{Primordial Deuterium}

The most metal-poor DLAs are a window not only on early stellar nucleosynthesis, but also on primordial nucleosynthesis in the first few minutes after the Big-Bang (BBN). In particular, such DLAs are the best astrophysical environments for the determination of the primordial abundance of deuterium, $(\mathrm{D} / \mathrm{H})_{\mathrm{p}}$, 'the baryometer of choice' in the words of David Schramm and Mike Turner [14]. Consider the following. For metallicities lower than 1/100 of solar, the corrections for the astration of deuterium (its destruction through cycles of star formation) are minimal. The most metal-poor DLAs are also the ones with the simplest internal kinematics; the absorption usually takes place in just one or two discrete components with internal velocity dispersion of only a few
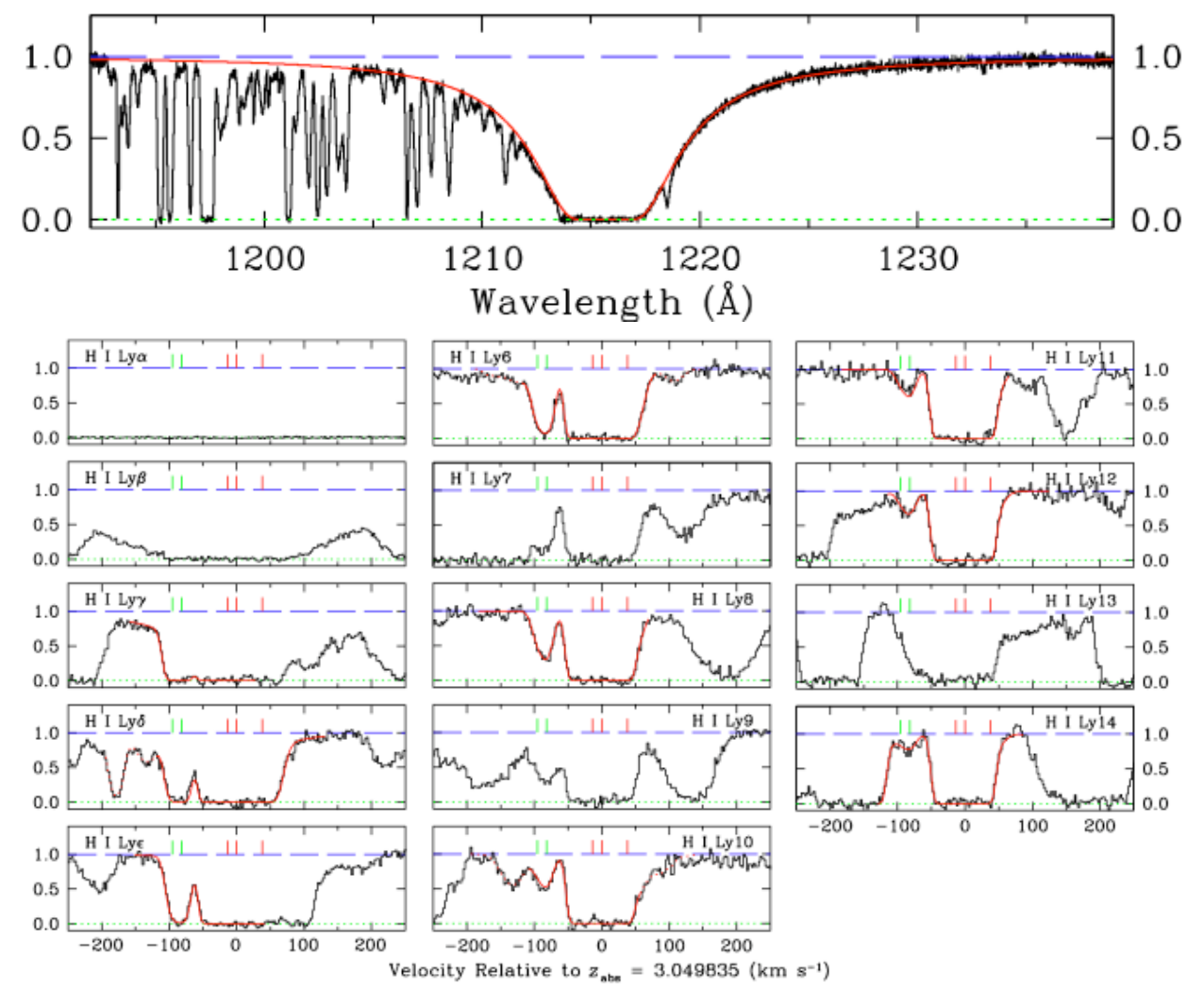

Figure 6: Top panel: The Ly $\alpha$ region in the UVES spectrum of SDSSJ1419+0829. The spectrum (black histogram) has been normalised to the QSO continuum and reduced to the rest-frame of the $z_{\mathrm{abs}}=3.04984$ DLA. The red line shows the best-fitting damped Ly $\alpha$ absorption profile, produced by a neutral hydrogen column density $\log N(\mathrm{HI}) / \mathrm{cm}^{-2}=20.391 \pm 0.008$. Bottom panels: Lyman series lines in the DLA. The black histogram is the observed spectrum, while the red continuous line is the model fit to the absorption features. Vertical tick marks above the spectrum indicate the three absorption components contributing to the H I (red) and D I (green) absorption. In all panels, the $y$-axis scale is residual intensity. 


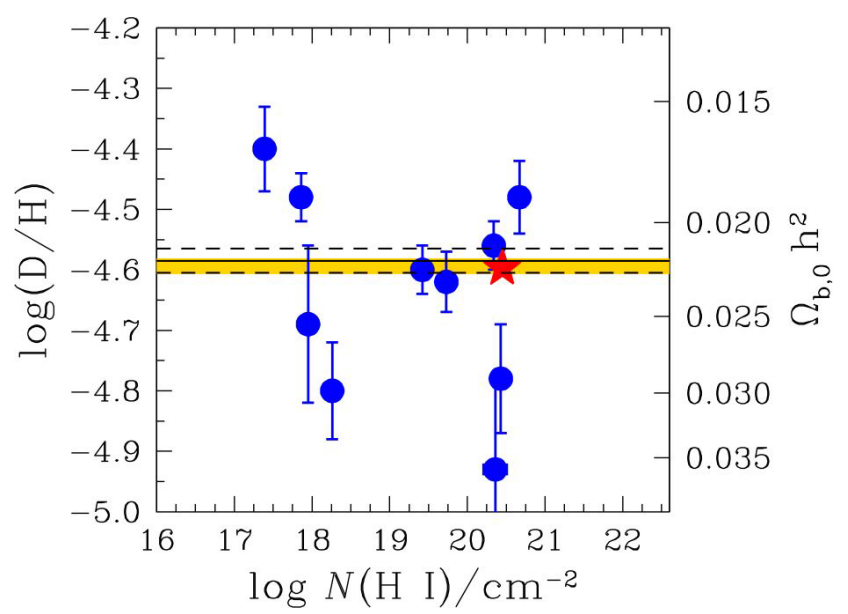

Figure 7: Measures of the deuterium abundance in high redshift QSO absorbers. Only cases were the deuterium absorption is clearly resolved from nearby spectral features are shown. The red star refers to the most recent measurement by Pettini \& Cooke [15], with errors smaller than the symbol size. The sources of earlier determinations are given in [15]. The horizontal lines are drawn at the weighted mean value of log $(\mathrm{D} / \mathrm{H})$ and its error, as determined with the bootstrap method. The yellow shaded area shows the range in $\Omega_{\mathrm{b}, 0} h^{2}(\mathrm{CMB})$ from [16].

$\mathrm{km} \mathrm{s}^{-1}$, allowing the D isotope shift of $-82 \mathrm{~km} \mathrm{~s}^{-1}$ to be resolved. Furthermore, the high column densities of neutral gas in DLAs make many absorption lines in the Lyman series of D I accessible to observation and measurement, greatly improving the accuracy with which the $\mathrm{D} / \mathrm{H}$ ratio can be determined, compared to other classes of QSO absorbers.

Reliable measures of $(\mathrm{D} / \mathrm{H})_{\mathrm{p}}$ are still scarce after nearly twenty years of high resolution spectroscopy with 8-10 m class telescopes, but our survey of VMP DLAs has the potential of significantly improving current statistics. One of our targets, the $z_{\mathrm{abs}}=3.04984$ DLA in the QSO $\mathrm{J} 1419+0829$, turns out to have near-ideal properties for an accurate determination of $(\mathrm{D} / \mathrm{H})_{\mathrm{p}}$. This realisation spurred us to develop software specifically designed to deduce the best fitting value of $\mathrm{D} / \mathrm{H}$ and to assess comprehensively the random and systematic errors affecting this determination [15]. In Figure 6 we have reproduced portions of the VLT-UVES spectrum of this QSO encompassing lines in the Lyman series of the DLA. D I absorption is clearly separated from nearby $\mathrm{H}$ I in eight transitions of widely different $f$-values, from $\operatorname{Ly} \delta$ to Ly 14 .

We find $\mathrm{D} / \mathrm{H}=(2.535 \pm 0.05) \times 10^{-5}$, where the uncertainty includes both random and systematic errors estimated via Monte Carlo techniques. This is the most precise estimate of $(\mathrm{D} / \mathrm{H})_{\mathrm{p}}$ to date (see Figure 7). It implies $\Omega_{\mathrm{b}, 0} h^{2}=0.0223 \pm 0.0009^{1}$, in very good agreement with $\Omega_{\mathrm{b}, 0} h^{2}(\mathrm{CMB})=0.0222 \pm 0.0004$ deduced from the analysis of the power spectrum of the temperature fluctuations of the cosmic microwave background [16].

The consistency between the cosmic density of baryons measured from entirely different physical processes operating, respectively, in the first few minutes of our Universe's existence and $\sim 375000$ years later gives confidence in the validity of the standard cosmological model. As has been discussed extensively in recent years (e.g. [18]), the comparison between $\Omega_{\mathrm{b}, 0}(\mathrm{BBN})$ and

\footnotetext{
${ }^{1}$ Note that the error has doubled from $2 \%$ to $4 \%$ in converting $(\mathrm{D} / \mathrm{H})_{\mathrm{p}}$ to $\Omega_{\mathrm{b}, 0} h^{2}$. The additional source of error is the difference between theoretical and experimental estimates of the nuclear reaction rate $d(p, \gamma)^{3} \mathrm{He}$ [17].
} 
$\Omega_{\mathrm{b}, 0}(\mathrm{CMB})$ can be used to put constraints on the number of sterile neutrinos (sometimes fashionably referred to as 'dark radiation') and on a possible lepton asymmetry. While these intriguing speculations have yet to be put to rest, we note here that what we consider to be the most accurate estimate of the primordial abundance of $\mathrm{D}$ limits the number of neutrino families to $\mathrm{N}_{v}=3.0 \pm 0.5$.

\section{Conclusions}

The role that DLAs can play in exploring the origin of chemical elements has become increasingly appreciated since the first efforts in this direction (e.g. [19]). The survey of the most metal-poor DLAs we have described here has resolved a number of issues lingering from analogous stellar studies. The enhancement of $\mathrm{O}$ relative to $\mathrm{Fe}$ is relatively modest, amounting to a factor of $\sim 2.5$ in the metallicity range $[\mathrm{Fe} / \mathrm{H}]=-2$ to -3 . It seems likely that the 'First Stars' were copious $\mathrm{C}$ producers. Our ideas about the relative importance of primary and secondary production of $\mathrm{N}$ appear to be approximately correct. The abundance of D measured in high redshift clouds confirms the fundamental framework of Big-Bang nucleosynthesis without recourse to non-standard physics.

As is always the case, these results whet our appetite for further questions. Does the Oenhancement increase at the lowest metallicities, as may be expected if the First Stars were very massive? Can we find further examples of CEMP DLAs, and can we constrain the IMF of the First Stars from their chemical composition? Can massive stars produce primary N, perhaps through rotation? Is it possible to improve the overall accuracy of $\Omega_{\mathrm{b}, 0}(\mathrm{BBN})$ and thereby place tighter constraints on non-standard physics in conjunction with the forthcoming measurements of $\Omega_{\mathrm{b}, 0}(\mathrm{CMB})$ from the Planck mission? We are confident that significant progress will have been made on at least some of these issues by the time we meet again at the next 'Nuclei in the Cosmos' meeting.

It is a pleasure to acknowledge our collaborators in the projects described in this conference presentation: Regina Jorgenson, Michael Murphy, Poul-Erik Nissen, Gwen Rudie, and Chuck Steidel. We are grateful to the time allocation panels of the Keck and VLT telescopes for their support of this demanding observational programme. Special thanks the organising committee of Nuclei in the Cosmos XII for running a smooth, engaging, and altogether very successful meeting.

\section{References}

[1] M. Pettini, A. Boksenberg, R.W. Hunstead, Metal enrichment, dust, and star formation in galaxies at high redshifts. I - The $z=2.3091$ absorber toward PHL 957, ApJ, 348, 48 (1990).

[2] S. Ellison et al., H I content, metallicities and spin temperatures of damped and sub-damped Ly $\alpha$ systems in the redshift desert, MNRAS, 424, 293 (2012).

[3] R. Cooke et al., The most metal-poor damped Ly $\alpha$ systems: insights into chemical evolution in the very metal-poor regime, MNRAS, 417, 1534 (2011).

[4] M. Asplund et al., The Chemical composition of the Sun, ARAA, 47, 481.

[5] P.S. Conti et al., Neutral Oxygen in late-type stars, ApJ, 148, 105 (1967).

[6] J.C. Wheeler, C. Sneden, J.W. Truran, Abundance ratios as a function of metallicity, ARAA, 27, 279. 
[7] C.J. Akerman et al., The evolution of the C/O ratio in metal-poor halo stars, A\&A, 414, 931 (2004).

[8] Fabbian et al., The C/O ratio at low metallicity: constraints on early chemical evolution from observations of Galactic halo stars, A\&A, 500, 1142 (2009).

[9] R. Cooke et al., A carbon-enhanced metal-poor damped Ly $\alpha$ system: probing gas from Population III nucleosynthesis?, MNRAS, 412, 1047 (2011).

[10] R. Cooke, M. Pettini, \& M.T. Murphy, A new candidate for probing Population III nucleosynthesis with carbon-enhanced damped Ly $\alpha$ systems, MNRAS, 425, 347 (2012).

[11] M. Pettini et al., C, N, O abundances in the most metal-poor damped Lyman alpha systems, MNRAS, 385, 2011 (2008).

[12] M.B. Vila-Costas \& Edmunds, M.G., The Nitrogen-to-Oxygen ratio in galaxies, and its implications for the origin of Nitrogen, MNRAS, 265, 199 (1993).

[13] R.B.C. Henry \& J.X. Prochaska, The chemical evolution of high-z galaxies from the relative abundances of N, Si, S, and Fe in damped Ly $\alpha$ systems, PASP, 119, 962 (2007).

[14] D.N. Schramm, \& M.S. Turner, Big-bang nucleosynthesis enters the precision era, RevModPhys, 70, 303 (1998).

[15] M. Pettini \& R. Cooke, A new, precise measurement of the primordial abundance of Deuterium, $M N R A S$, in press (2012).

[16] R. Keisler et al., A measurement of the damping tail of the cosmic microwave background power spectrum with the South Pole telescope, ApJ, 743, 28 (2011).

[17] K.M. Nollett \& G.P. Holder, An analysis of constraints on relativistic species from primordial nucleosynthesis and the cosmic microwave background, Phys. Rev. D, in press, eprint arXiv: 1112.2683 (2011).

[18] G. Steigman, Neutrinos And Big Bang Nucleosynthesis, eprint arXiv:1208.0032 (2012).

[19] M. Pettini, K. Lipman, \& R.W. Hunstead, Element Abundances at High Redshifts: The N/O Ratio in a Primeval Galaxy, ApJ, 451, 100 (1995).

\section{Questions}

Jennifer Johnson: What are the sizes and nature of the systems that you are probing and can that tell us anything about mixing in the early Galaxy?

Max Pettini: It is a source of considerable frustration that, while we can study in great detail the physical properties of these absorbers from high resolution spectroscopy, it has proved very difficult to associate them with particular classes of galaxies. Imaging searches for their optical counterparts have so far shown that a wide range of galaxies, spanning many magnitudes in the galaxy luminosity function, can give rise to DLAs. My instinct tells me that the most metal-poor DLAs, on which I have concentrated today, may also be the most difficult to image directly, if they have indeed experienced very low levels of star formation as their chemically unevolved status suggests.

Chiaki Kobayashi: Can you comment on other problems in observations eg. dust depletion and temperature effect? 
Max Pettini: Dust depletions are very much reduced with decreasing metallicities and are expected to make only negligible corrections to the element abundances deduced from the gas-phase in VMP DLAs. We can get a good handle on the temperature of the gas by comparing the widths of absorption lines from elements of different masses. Temperatures of a few thousand degrees are indicated for the DLAs I have focussed on today.

Taka Kajino: The analysis of WMAP7 data of CMB anisotropies alone gives us $\mathrm{Y}_{\mathrm{p}} \geq 0.3$ and $\mathrm{N}_{v} \geq 5$, which are too large to accept. Is it possible to detect ${ }^{4} \mathrm{He}$ abundance from Lyman-alpha clouds as you did for D?

Max Pettini: I wish it were possible! But the He I lines all occur at very short wavelengths, where the Universe is opaque to EUV radiation. In the words of the sage: "Never say never again", but the difficulties are enormous.

Harriet Dinerstein: You mentioned that the C-enhanced DLAs may be the counterparts of the CEMP-no stars. Do you have any upper limits on neutron-capture elements in these systems?

Max Pettini: This is a very good point, Harriet. To really substantiate that statement we would need to measure the abundances of neutron-capture elements, a task for the 30-m class telescopes of the future.

Gabriele Cescutti: What are the results concerning nitrogen in DLA systems?

Max Pettini: I didn't have sufficient time to discuss nitrogen in my oral presentation, but I have included the results you are interested in in Section 4 of this conference report. 